\title{
Evidence for interplay among yeast replicative DNA polymerases alpha, delta and epsilon from studies of exonuclease and polymerase active site mutations Youri I Pavlov*1,2, Satoko Maki ${ }^{3}$, Hisaji Maki ${ }^{3}$ and Thomas A Kunkel1,4
}

\begin{abstract}
Address: ${ }^{1}$ Laboratory of Molecular Genetics, National Institute of Environmental Health Sciences, National Institute of Health, DHHS, Research Triangle Park, NC 27709, USA, ${ }^{2}$ Eppley Institute for Research in Cancer and Department of Biochemistry and Molecular Biology, University of Nebraska Medical Center, Omaha, NE 68198, USA, ${ }^{3}$ Laboratory of Microbial Molecular Genetics, Graduate School of Biological Sciences, Nara Institute of Science and Technology, Nara 630-01, Japan and ${ }^{4}$ Laboratory of Structural Biology, National Institute of Environmental Health Sciences National Institute of Health, DHHS, Research Triangle Park, NC 27709, USA
\end{abstract}

Email: Youri I Pavlov* - ypavlov@unmc.edu; Satoko Maki - smaki@bs.naist.jp; Hisaji Maki - maki@bs.naist.jp; Thomas A Kunkel - kunkel@niehs.nih.gov

* Corresponding author

Published: 26 May 2004

BMC Biology 2004, 2:II
Received: 16 January 2004

Accepted: 26 May 2004

This article is available from: http://www.biomedcentral.com/I74I-7007/2/II

(c) 2004 Pavlov et al; licensee BioMed Central Ltd. This is an Open Access article: verbatim copying and redistribution of this article are permitted in all media for any purpose, provided this notice is preserved along with the article's original URL.

\begin{abstract}
Background: DNA polymerase $\varepsilon(\mathrm{Pol} \varepsilon)$ is essential for S-phase replication, DNA damage repair and checkpoint control in yeast. A pol2-Y83 IA mutation leading to a tyrosine to alanine change in the Pol $\varepsilon$ active site does not cause growth defects and confers a mutator phenotype that is normally subtle but strong in a mismatch repair-deficient strain. Here we investigate the mechanism responsible for the mutator effect.
\end{abstract}

Results: Purified four-subunit Y83IA Pol $\varepsilon$ turns over more deoxynucleoside triphosphates to deoxynucleoside monophosphates than does wild-type Pol $\varepsilon$, suggesting altered coordination between the polymerase and exonuclease active sites. The pol2-Y83IA mutation suppresses the mutator effect of the pol2-4 mutation in the exonuclease active site that abolishes proofreading by Pol $\varepsilon$, as measured in haploid strain with the pol2-Y83/A,4 double mutation. Analysis of mutation rates in diploid strains reveals that the pol2-Y83/A allele is recessive to pol2-4. In addition, the mutation rates of strains with the pol2-4 mutation in combination with active site mutator mutations in Pol $\delta$ and Pol $\alpha$ suggest that Pol $\varepsilon$ may proofread certain errors made by Pol $\alpha$ and Pol $\delta$ during replication in vivo.

Conclusions: Our data suggest that Y83 IA replacement in Pol $\varepsilon$ reduces replication fidelity and its participation in chromosomal replication, but without eliminating an additional function that is essential for viability. This suggests that other polymerases can substitute for certain functions of polymerase $\varepsilon$.

\section{Background}

Multiple DNA polymerases are thought to be present at the eukaryotic replication fork [1-4]. Some of their functions could be unique while others could be overlapping. Different polymerases may compete for certain DNA sub- strates and several polymerases may sometimes act in concert [5-9]. Under normal circumstances, chromosomal replication requires at least three DNA polymerases, $\operatorname{Pol} \alpha$, Pol $\varepsilon$ and Pol $\delta$. All of these polymerases are multi-subunit complexes $[1,4]$ and all subunits are required for their 
proper function (see recent papers [10-12], and references therein). Pol $\alpha$ is not very processive and lacks an intrinsic proofreading exonuclease. It has a tightly associated activity for the synthesis of RNA primers at replication origins and on the lagging DNA strand. Pol $\alpha$ extends these RNA primers by synthesizing short stretches of DNA, and then a switch occurs to processive synthesis by Pol $\varepsilon$ and/or Pol $\delta$.

The exact roles of Pol $\varepsilon$ and Pol $\delta$ in replication are not yet fully understood. Among several possible models, it has been proposed that Pol $\varepsilon$ is primarily responsible for copying the leading strand DNA template and $\operatorname{Pol} \delta$ is responsible for lagging strand replication [13]. Another model has proposed the opposite [14]. Either model is consistent with the fact that Pol $\varepsilon$ and Pol $\delta$ both possess intrinsic 3' to $5^{\prime}$ exonuclease activity, and with genetic data in yeast suggesting that these nucleases proofread replication errors on opposite DNA strands during chromosomal [15] or plasmid [16] DNA replication. However, the replication functions of $\operatorname{Pol} \delta$ and $\operatorname{Pol} \varepsilon$ are not equivalent [17]. When proofreading or base selectivity is impaired by homologous active site point mutations in POL3 (encoding Pol $\delta$ ) and POL2 (encoding Pol $\varepsilon$ ), the mutator effects are much stronger for pol3 mutants than for pol2 mutants $[7,18,19]$. A yeast strain with an amino-terminal deletion of the polymerase domain of the POL2 gene but retaining the carboxyl-terminal domain grows slowly but is nonetheless viable $[20,21]$, and references therein), indicating that another polymerase can substitute for the polymerization function of Pol $\varepsilon$. Thus, it is possible that Pol $\delta$ may perform the bulk of chain elongation during chromosomal replication [17], while Pol $\varepsilon$ serves more specialized roles. One possibility is a role in the $\mathrm{S}$ phase checkpoint control when replication fork progression is impeded [22], perhaps by sensing single-stranded DNA [23]. Pol $\varepsilon$ also interacts with Dpb11 and, thus, may function during initiation of DNA replication at origins $[24,25]$. It has also been suggested that Pol $\varepsilon$ may participate in replication during late, but not early, S phase [26], as well as in the establishment of sister chromatid cohesion [27].

The fidelity of nuclear DNA replication in eukaryotic cells relies on three steps that are thought to operate in series: the base selectivity of DNA polymerases that operate at the fork, proofreading by the exonucleases of DNA polymerases $\varepsilon$ and $\delta$ or possibly by extrinsic exonucleases, and DNA mismatch repair. This is supported genetically by the fact that the double mutants that are deficient in proofreading by either DNA polymerase and mismatch repair are hypermutable, suggesting a sequential action of proofreading and mismatch repair $[19,28]$. As one attempt to understand the complex enzymology that determines fidelity and influences eukaryotic genome stability, we recently described the effects on viability and mutagenesis in Saccharomyces cereivisiae resulting from replacing a conserved tyrosine in the active sites of three replicative polymerases with alanine [7]. Important for our understanding of the role of Pol $\varepsilon$, a haploid yeast strain with this pol2-Y831A mutation grew normally, suggesting no major defects in replication. However, we observed a modest, spontaneous base substitution mutator effect and a strong, spontaneous frameshift mutator effect when DNA mismatch repair was nonfunctional. These mutator effects suggested a defect in replication fidelity at a step preceding mismatch repair (for example, reduced polymerase base selectivity and/or proofreading) [7]. The pol2-Y831A mutation was semi-dominant in heterozygous diploid cells, suggesting that the mutator effects reflected an aberrant function of the altered Pol $\varepsilon$ that is not masked by the presence of wild-type Pol $\varepsilon$. Here we further investigate the mutator effects conferred by the pol2-Y831A mutation using biochemical and genetic approaches. The results suggest decreased participation of Y831A Pol $\varepsilon$ in chromosomal replication. We also investigate the possibility that wild-type Pol $\varepsilon$ proofreads replication errors generated by altered variants of DNA polymerases $\alpha$ and $\delta$.

\section{Results \\ Biochemical characterization of Y83 IA Pol $\varepsilon$}

We purified the four-subunit DNA polymerase $\varepsilon$ from a yeast strain with a chromosomal pol2-Y831A mutation (Figure 1). The quality of the preparation, in terms of purity and specific activity, was comparable to the wildtype polymerase $\varepsilon$ that we purified previously by the same method; exonuclease activity co-eluted with polymerase activity $[23,29]$. We compared the polymerase and exonuclease activity of the current preparation with DNA polymerase $\varepsilon$ purified earlier from a wild-type strain. We first compared turnover of deoxythymidine triphosphate (dTTP) to deoxythymidine monophosphate (dTMP) during DNA synthesis by wild-type and mutant polymerases on poly $(\mathrm{dA})$ template by thin-layer chromatography (TLC) (Figure 2A,2B). This method enables simultaneous measurement and comparison of exonuclease and DNA polymerase activity. dTMP turnover rates, ((pmols dTMP excised $) /($ pmols dTMP incorporated + excised $) \times 100)$, for wild-type and the mutant polymerases were $22 \%$ and $50 \%$, respectively (Figure 2C,2D). The value for the mutant Pol $\varepsilon$ was unchanged, even after further purification of the enzyme preparation using a gel-filtration column chromatography, suggesting that the high turnover number feature is intrinsic to the mutant Pol $\varepsilon$. In further experiments, when the exonuclease activity was assayed separately with single-stranded DNA as a substrate (see Methods), both enzyme preparations had the same ratio of 3 ' to 5 ' exonuclease activity to polymerase activity, 3.7 for wild-type and 3.5 for Pol $\varepsilon$ Y831A. This suggests that the increased rate of dTMP turnover by the mutant 


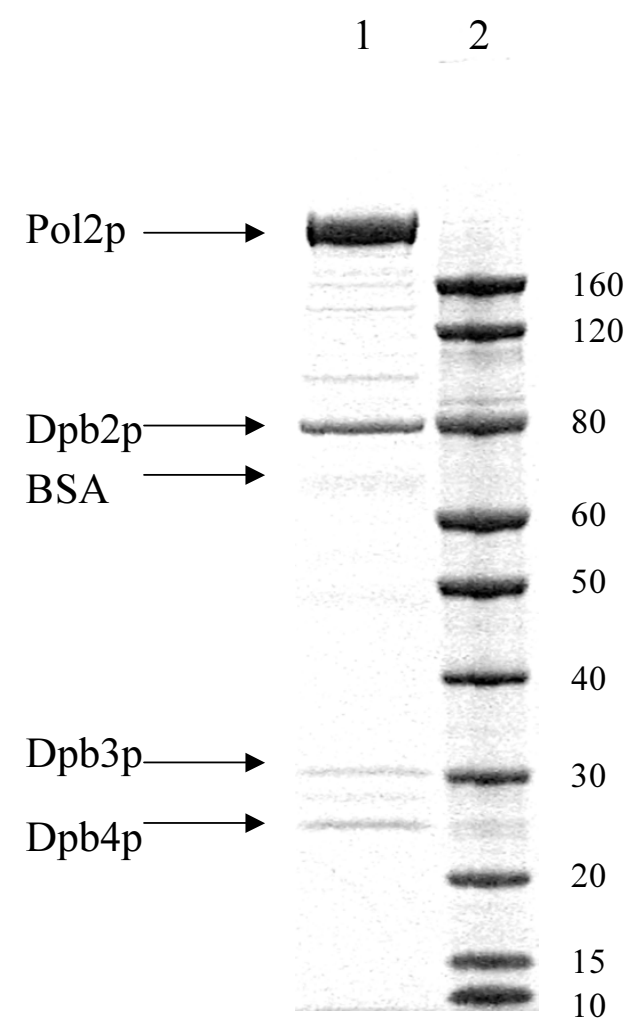

\section{Figure I}

Purified four-subunit Y83 IA Pol $\varepsilon$. SDS-PAGE analysis of peak fraction from final SMART MonoS column (see Methods). I $\mu$ l of collected fraction 15 was mixed with Invitrogen loading buffer and run on denaturing 4-I2\% NUPAGE Bis-Tris polyacrylamide gel for 50 minutes at $200 \mathrm{~V}$ in MOPS buffer. The gel was stained with SimpleStain colloidal blue stain as recommended by the vendor (Invitrogen). Lane I, Pol $\varepsilon$ Y83 IA; lane 2, the Benchmark His-tagged protein standards (Invitrogen).

polymerase is not due to an alteration in the catalytic efficiencies of polymerase and proofreading exonuclease activities, but rather that the tyrosine to alanine substitution in the polymerase active site alters communication between the polymerase and exonuclease active sites.

\section{Mutation rates in haploids with double pol2-Y83 I,4 mutation}

Strains with the pol2-Y831A mutation are mutators in a mismatch repair defective background [7], suggesting that they are defective in controlling replication fidelity at a step prior to mismatch repair (MMR). If this defect is due to an altered base selectivity of Y831A Pol $\varepsilon$, the resulting mismatches could be proofread by the intrinsic 3' exonuclease activity of Pol $\varepsilon$. In this case, mutational inactivation of the exonuclease should elevate mutation rates. Such a synergistic increase in mutation rate was previously observed in a strain with pol2-4,C1089 double mutation that inactivates proofreading and alters the polymerase domain [30]. To test this possibility with the pol2-Y831A mutant, we constructed haploid yeast strains with either single or double mutations in the POL2 gene, one in the polymerase active site (pol2-Y831A) and another in the exonuclease active site (pol2-4). These are derivatives of the previously developed strain 8C-YUNI101, which allows concomitant measurement of mutation rates at 
A.

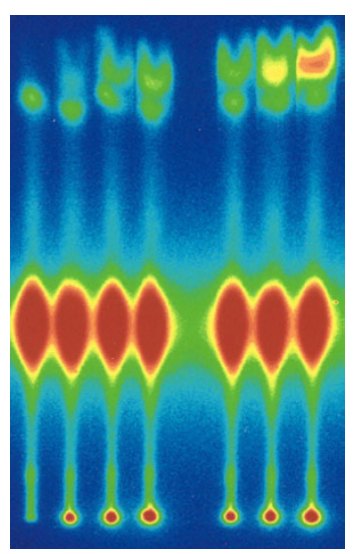

$\begin{array}{lllllll}1 & 2 & 3 & 4 & 5 & 6 & 7\end{array}$
B.

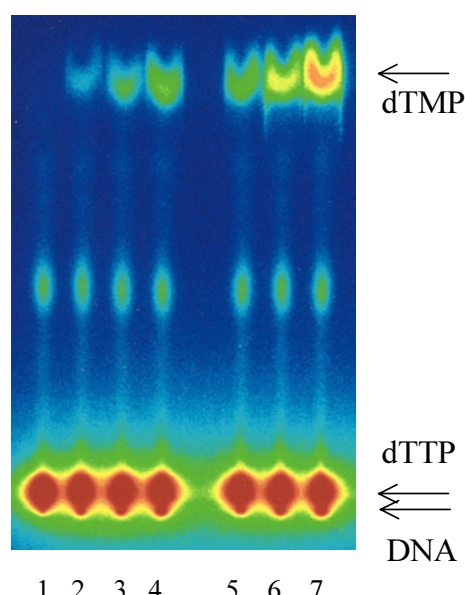

D.

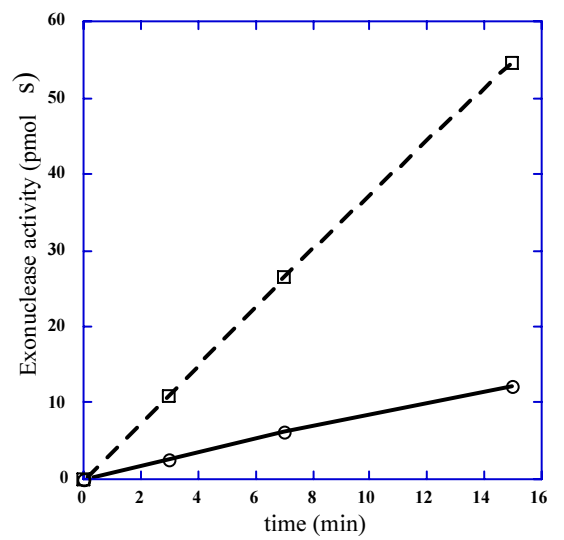

\section{Figure 2}

Elevated nucleotide turnover by Y83 IA Pol $\varepsilon$. DNA synthesis and dTTP turnover by Pol $\varepsilon$ was measured on poly(dA)/oligo(dT) substrate. Reactions were performed as described in Methods, using $0.13 \mathrm{U}$ of each enzyme for $20 \mu \mathrm{l}$ reactions. (A) Analysis of polymerase reaction by TLC in I M LiCl running buffer. Lanes I, 2, 3, 4: wild-type Pol $\varepsilon$ at $0,3,7$ and I5 minutes of reaction, respectively. Lanes 5, 6, 7: reactions with Y83IA Pol $\varepsilon$ at 3, 7 and I5 minutes, respectively. Positions of unincorporated label, label in DNA and dTMP are shown by arrows. (B) Analysis of polymerase reaction by TLC in $0.4 \mathrm{M} \mathrm{LiCl}$ running buffer. Lane assignment is the same as in (A). (C) Plot of time-course of DNA synthesis and DTMP turnover by wild-type Pol $\varepsilon$. Open circles connected by solid line represent DTMP retained into DNA; open rectangles connected by a dashed line represent excised dTMP. (D) Plot of time-course of DNA synthesis and dTMP turnover by Y83 IA Pol $\varepsilon$. Symbols are the same as in (C). 
Table I: Effect of combining the exonuclease and active site mutations in Pol $\varepsilon$ gene on mutation rates in haploids in $8 \mathrm{C}-Y$ YNII0 I genetic background

\begin{tabular}{|c|c|c|c|}
\hline \multirow[t]{3}{*}{ POL2 allele } & \multicolumn{3}{|c|}{ Mutation rates for different markers* } \\
\hline & \multirow[t]{2}{*}{ Forward mutation } & \multicolumn{2}{|c|}{ Reversion } \\
\hline & & Base substitutions & Frameshifts $(+I)$ \\
\hline & $\operatorname{Can}^{r}\left(\times 10^{-7}\right)$ & $\mathrm{Ura}^{+} \times\left(10^{-8}\right)$ & $\mathrm{His}^{+} \times\left(10^{-8}\right)$ \\
\hline \multicolumn{4}{|c|}{ Mismatch repair proficient strains } \\
\hline \multirow[t]{2}{*}{ wild-type } & 3.3 & 1.3 & 1.1 \\
\hline & $2.5-4.9$ & $1.1-1.7$ & $0.9-3.5$ \\
\hline \multirow[t]{2}{*}{ pol2-Y83IA } & 5.1 & 1.2 & 1.6 \\
\hline & $3.5-12.1$ & $0.8-2.8$ & $0.5-2.7$ \\
\hline \multirow[t]{2}{*}{ pol2-4 } & 27 & $3.4^{1}$ & $45^{2}$ \\
\hline & $15-42$ & $2.4-5.7$ & $35-78$ \\
\hline \multirow[t]{2}{*}{ pol2-4,Y83 IA } & 9 & 1.5 & $3.9^{3}$ \\
\hline & $7-15$ & $1.1-1.9$ & $2.3-5.4$ \\
\hline \multicolumn{4}{|c|}{ Mismatch repair deficient strains (pmsl) } \\
\hline \multirow[t]{2}{*}{ wild-type } & 90 & 2.8 & 87 \\
\hline & $72-150$ & $2.4-4.1$ & $54-150$ \\
\hline \multirow[t]{2}{*}{ pol2-Y83IA } & 340 & $12^{4}$ & $470^{5}$ \\
\hline & $230-420$ & $11-19$ & $270-790$ \\
\hline \multirow[t]{2}{*}{ pol2-4 } & 760 & $160^{6}$ & $1600^{7}$ \\
\hline & $120-1060$ & $90-420$ & $950-2800$ \\
\hline \multirow[t]{2}{*}{ pol2-4,Y83 IA } & 270 & $15^{8}$ & $410^{9}$ \\
\hline & $190-440$ & $8-55$ & $260-440$ \\
\hline
\end{tabular}

*Here and thereafter, median for 9-27 independent cultures with $95 \%$ confidence limits below. $1 / 4 / 16$ of revertants sequenced were due to C->A transversions $220 / 20$ revertants sequenced contained a $+\mid A$ frameshift event ${ }^{3} / 6 / 16$ revertants sequenced were due to $+\mid A$ frameshift ${ }^{4} / 2 / 14$ revertants were due to $C$ - $>A$ change $5 / 5 / I 5$ revertants sequenced were due to $+\mid A$ frameshift $6 / 2 / I 2$ revertants sequenced were due to $C$ - $>A$ mutation ${ }^{7} / 6 / 16$ revertants sequenced were due to $+I A$ frameshift $8 / 2 / 15$ revertants sequenced were due to $C->A$ mutation $9 / 4 / 14$ revertants were due to + IA frameshift

Table 2: Effect of combining the exonuclease and active site mutations in DNA polymerase $\varepsilon$ on mutation rates in diploids. Mismatch repair proficient strains

\begin{tabular}{|c|c|c|}
\hline Relevant mutation & $\mathrm{His}^{+}$reversion rate $\left(\times 10^{-8}\right)$ & Fold increase \\
\hline \multirow[t]{2}{*}{$+/+$} & 0.5 & 1 \\
\hline & $0.3-1.2$ & \\
\hline \multirow[t]{2}{*}{ +/ pol2-Y83 IA } & 1.1 & 2.2 \\
\hline & $0.7-1.7$ & \\
\hline \multirow[t]{2}{*}{ pol2-Y83IA/ pol2-Y83IA } & 2.2 & 4.4 \\
\hline & I.8-5.7 & \\
\hline \multirow[t]{2}{*}{$+/ p o l 2-4$} & 3.8 & 7.6 \\
\hline & $2.6-4.7$ & \\
\hline \multirow[t]{2}{*}{ pol2-4/pol2-4 } & 26 & 52 \\
\hline & $16-45$ & \\
\hline \multirow[t]{2}{*}{ pol2-4/ pol2-Y83IA } & 28 & 56 \\
\hline & $16-53$ & \\
\hline \multirow[t]{2}{*}{ pol2-4/ pol2-4,Y83 IA } & 21 & 42 \\
\hline & $18-34$ & \\
\hline \multirow[t]{2}{*}{ pol2-Y83 IA/ pol2-4,Y83 IA } & 1.8 & 3.6 \\
\hline & $1.2-3.1$ & \\
\hline \multirow[t]{2}{*}{ pol2-4,Y83 IA/pol2-4,Y83 IA } & 1.8 & 3.6 \\
\hline & $1.1-2.3$ & \\
\hline
\end{tabular}


Table 3: Effect of combining the exonuclease and active site mutations in DNA polymerase $\varepsilon$ on mutation rates in diploids. Mismatch repair deficient strains ( $p m s / / p m s I)$

\begin{tabular}{|c|c|c|}
\hline Relevant mutation & $\mathrm{His}^{+}$reversion rate $\left(\times 10^{-8}\right)$ & Fold increase \\
\hline \multirow[t]{2}{*}{$+/+$} & 110 & 1 \\
\hline & $90-140$ & \\
\hline \multirow[t]{2}{*}{$+/ p o l 2-Y 83 / A$} & 400 & 4 \\
\hline & $270-600$ & \\
\hline \multirow[t]{2}{*}{ pol2-Y83 IA/pol2-Y83 IA } & 820 & 7 \\
\hline & $750-950$ & \\
\hline \multirow[t]{2}{*}{$+/$ pol2-4 } & 1,500 & 14 \\
\hline & $930-3600$ & \\
\hline \multirow[t]{2}{*}{ pol2-4/pol2-4 } & 9,400 & 85 \\
\hline & $5,900-14,000$ & \\
\hline \multirow[t]{2}{*}{ pol2-4/ pol2-Y83IA } & 8,400 & 76 \\
\hline & $3,500-17,000$ & \\
\hline \multirow[t]{2}{*}{ +/pol2-4,Y83 IA } & 210 & 2 \\
\hline & $180-270$ & \\
\hline \multirow[t]{2}{*}{ pol2-4/pol2-4,Y83 IA } & $\mathrm{II}, 000$ & 100 \\
\hline & $8,100-22,000$ & \\
\hline \multirow[t]{2}{*}{ pol2-Y83 IA/pol2-4,Y83IA } & 1,200 & 11 \\
\hline & $1,030-1,460$ & \\
\hline \multirow[t]{2}{*}{ pol2-4,Y83 IA/pol2-4,Y83 IA } & 1,020 & 9 \\
\hline & $840-2,300$ & \\
\hline
\end{tabular}

several loci. These include the forward mutation rate at the CAN1 locus, where mutations reflect a variety of substitution, frameshift and more complex events; the rate of reversion of the ura3-29 missense mutation which reverts via three specific base pair substitutions at a single $\mathrm{G} \rightarrow \mathrm{C}$ base pair in a TCT codon encoding for serine [7]; and reversion of the his7-2 mutant allele, which occurs mainly via +1 frameshifts in a homopolymeric $A \rightarrow T$ run $[7,31]$. In a MMR-proficient background (Table 1, top), the pol2Y831A mutation results in a slight or no mutator effect, while loss of proofreading by Pol $\varepsilon$ elevates the mutation rate at CAN1, ura3-29 and his7-2 by eight-fold, 2.6-fold and 41-fold, respectively. In an MMR-deficient background (Table 1, bottom), the pol2-Y831A mutation and the pol2-4 mutation each elevates the mutation rate at all three loci. Surprisingly, when both mutations are present in the same gene, the pol2-Y831A mutation strongly suppresses the mutator effects of the pol2-4 mutation for all three markers tested in both MMR-proficient and MMRdeficient strains. Thus, despite generating a mutator effect itself, pol2-Y831A actually suppresses mutations resulting from loss of proofreading by Pol $\varepsilon$. This suppression suggests that Y831A Pol $\varepsilon$ may not participate in replication to the same extent as does wild-type Pol $\varepsilon$.

To further test this hypothesis, we measured $\mathrm{His}^{+}$reversion rates in a series of diploid strains. In MMR-proficient diploid strains (Table 2), the pol2-Y831A allele appeared to be semi-dominant, since the mutator effect of the het- erozygote was half that of the homozygote. This may be due to either effective competition of error-prone mutant polymerase with the wild-type enzyme or due to perturbations in DNA synthesis caused by Y831A Pol $\varepsilon$. The heterozygous +/pol2-4 and homozygous pol2-4/pol2-4 diploid strains had a reversion rate elevated by 7.6 -fold and 52 fold, respectively, suggesting that wild-type Pol $\varepsilon$ may efficiently compete with the exonuclease-deficient enzyme and proofread replication errors. When one chromosome possessed the pol2-4 allele while the other chromosome had the either pol2-Y831A alone or pol2-4,Y831A double mutation, the reversion rate was similar to the homozygous pol2-4/pol2-4 diploid strain. However, homozygosity for pol2-Y831A suppressed the mutator effect of the pol2-4 mutation, slightly in the +/pol2-4 diploid (two-fold, Table 2, penultimate line) and strongly in the pol2-4/pol2-4 diploid strain (14-fold, Table 2, last line). Similar effects were obtained in diploid strains in an MMR-deficient background (Table 3). These results are consistent with the results in the haploid strains, and further support the hypothesis that Y831A Pol $\varepsilon$ does not compete with wild-type exonuclease-deficient Pol $\varepsilon$.

\section{Lack of genetic interaction between pol2-Y83 I A and pol3-0 I mutation in exonuclease domain of Pol $\delta$}

The idea of altered communication between the polymerase and exonuclease active sites (see Figure 2) and the putative inability of Y831A Pol $\varepsilon$ to compete with exonuclease-deficient Pol $\varepsilon$ (Tables 1, 2 and 3) suggest that 
Table 4: Effect of combining the mutation in the exonuclease domain of the Pol $\delta$ gene with the active site mutation in Pol $\varepsilon$ genes on mutation rates in mismatch repair-proficient derivatives of $8 \mathrm{C}$-YUNII $0 \mathrm{I}$ strain

\begin{tabular}{|c|c|c|c|}
\hline \multirow[t]{3}{*}{ Relevant genotype } & \multicolumn{3}{|c|}{ Mutation rates for different markers } \\
\hline & \multirow{2}{*}{$\begin{array}{l}\text { Forward mutation } \\
\text { Can }^{r} \times\left(10^{-7}\right)\end{array}$} & \multicolumn{2}{|c|}{ Reversion } \\
\hline & & Base substitutions $\mathrm{Ura}^{+}\left(\times 10^{-8}\right)$ & Frameshifts $(+\mathrm{I}) \mathrm{His}^{+}\left(\times \mathrm{I0}^{-8}\right)$ \\
\hline \multirow[t]{2}{*}{ wild-type } & 2.9 & 1.5 & 1.5 \\
\hline & $1.6-4.9$ & $1.1-2.3$ & $1.2-2.1$ \\
\hline \multirow[t]{2}{*}{$+|p o l 3-0| *$} & 28 & $\mathrm{n} / \mathrm{a}$ & 33 \\
\hline & $22-28$ & & $31-39$ \\
\hline \multirow[t]{2}{*}{$+/$ pol3-0I*pol2-Y83IA } & 31 & $\mathrm{n} / \mathrm{a}$ & 27 \\
\hline & $25-39$ & & $24-34$ \\
\hline \multirow[t]{2}{*}{ pol3-0I } & 190 & 19 & 110 \\
\hline & $140-260$ & $6-35$ & $80-160$ \\
\hline \multirow[t]{2}{*}{ pol3-0I pol2-Y83IA } & 130 & 14 & 34 \\
\hline & $90-210$ & $13-28$ & $18-43$ \\
\hline
\end{tabular}

$\mathrm{n} / \mathrm{a}$, non applicable, since these strains are Ura ${ }^{+}$. Haploids with duplication of the POL3 gene.

Y831A Pol $\varepsilon$ may not edit its errors using its intrinsic proofreading exonuclease. In this case, a different exonuclease-proficient DNA polymerase may proofread errors by Y831A Pol $\varepsilon$. The precedent for this hypothesis comes from both in vitro [32] and in vivo [6] experiments indicating that the exonuclease activity of Pol $\delta$ can proofread errors by other replicative polymerases. To test if there is an effect of proofreading by Pol $\delta$ on the mutation rate in the pol2-Y831A mutant strain, we constructed strains with a combination of the pol2-Y831A mutation and the pol301 mutation that inactivates proofreading by Pol $\delta$. If the exonuclease activity of Pol $\delta$ is involved, a synergistic increase in mutation rate is expected in the double mutant. In a haploid strain with duplication of the POL3 gene, encoding a catalytic subunit of Pol $\delta$, when one allele is the pol3-01 and another allele is the wild-type, there is 9.6-fold increase in $\mathrm{Can}^{\mathrm{r}}$ forward mutation rate and a 22-fold increase in frameshift mutation rate (Table 4). A haploid strain with the pol3-01 mutation showed a 72-fold increase in the $\mathrm{Can}^{\mathrm{r}}$ mutation rate, a 15 -fold increase in the rate of ura3-29 reversion and a 73-fold increase in frameshift mutation rate. These results are consistent with the published data that the pol3-01 is a strong mutator allele that is semi-dominant in respect to the wild-type allele $[6,19]$. The mutation rates at these loci are no higher in the same strains containing additional pol2Y831A mutation (Table 4). According to our previous data, the synergistic interactions are readily detected with the pol2-Y831A allele, which is a weak mutator, as exemplified by an increase of mutation rates in the pol2-Y831A pms1 double mutants [7]. We conclude that errors made by Y831A Pol $\varepsilon$ are not proofread by Pol $\delta$.
Genetic interaction between active site mutations in genes encoding Pol $\alpha$ and Pol $\delta$ and proofreading defective mutations in POL2 and POL3

Although errors made by Y831A Pol $\varepsilon$ do not appear to be proofread by Pol $\delta$, it is possible that Pol $\varepsilon$ might proofread errors by other polymerases. To investigate this and to determine whether the effects of combining exonuclease-defective alleles of POL2 (Table 2) and POL3 (Table 4) with a polymerase active site defect were specific to Y831A Pol $\varepsilon$, we looked for genetic interaction between proofreading and mutator alleles of the POL1 (encoding Pol $\alpha$ ) and the POL3 (encoding Pol $\delta$ ). For this we chose the pol1-Y869A and pol3-Y708A mutations that lead to conserved tyrosine in the active site of Pol $\alpha$ (in region II) and Pol $\delta$ (in region III, in the same place as Tyr 831 in Pol $\varepsilon)$ to change to alanine. The pol1-Y869A mutation results in a weak mutator phenotype in a haploid yeast strain (Table 5, line 2). However, in marked contrast to decreased mutation rates with the pol2-Y831A mutation in the POL2, when the pol1-Y869A mutation is combined with the pol2-4 mutation, the mutation rates at the CAN1 and his7-2 loci are increased. The increase at the CAN1 locus is multiplicative rather than additive (Table 5, compare line 4 to lines 2 plus 3 ), and the increase at the his72 locus is intermediate between multiplicative and additive. The interaction between the pol1-Y869A mutation and the pol3-01 mutation that inactivates proofreading by Pol $\delta$ is somewhat different. In that case, the increases in mutation rates at the CAN1 and his7-2 loci are additive rather than multiplicative (Table 5, compare line 6 to lines 2 plus 5). This suggests that errors arising in the pol1Y869A mutant strain may be proofread by the intrinsic exonuclease activity of Pol $\varepsilon$, but not by the exonuclease activity of Pol $\delta$. Finally, when the Pol $\delta$ mutator allele 
Table 5: Effect of combining the mutation in exonuclease domains of DNA polymerases $\delta$ and $\varepsilon$ genes with active site mutation in DNA polymerases $\alpha$ and $\delta$ genes on mutation rates in mismatch repair proficient haploid derivatives of 8C-YUNIIOI

\begin{tabular}{|c|c|c|c|c|c|c|}
\hline \multirow[t]{3}{*}{ Relevant mutation } & \multicolumn{6}{|c|}{ Mutation rates } \\
\hline & \multicolumn{2}{|c|}{ Forward Can $r$ mutation } & \multicolumn{2}{|c|}{ Ura $^{+}$reversion (base substitutions) } & \multicolumn{2}{|c|}{$\mathrm{His}^{+}$reversion (+ I frameshifts) } \\
\hline & Rate $\left(\times 10^{-7}\right)$ & Fold increase & Rate $\left(\times 10^{-8}\right)$ & Fold increase & Rate $\left(\times 10^{-8}\right)$ & Fold increase \\
\hline \multirow[t]{2}{*}{ no } & 4.4 & I & 1.3 & I & 1.9 & 1 \\
\hline & $2.9-5.8$ & & $1.2-1.7$ & & $1.4-3.6$ & \\
\hline \multirow[t]{2}{*}{ poll-Y869A } & 12 & 2.7 & 1.1 & 0.8 & 6.4 & 3.3 \\
\hline & $6.2-28$ & & $0.8-2.2$ & & $4.1-16$ & \\
\hline \multirow[t]{2}{*}{ pol2-4 } & 17 & 3.8 & 5.1 & 3.9 & 51 & 27 \\
\hline & $12-24$ & & $3.7-5.5$ & & $45-71$ & \\
\hline \multirow[t]{2}{*}{ pol2-4 poll-Y869A } & 46 & 10 & 8.8 & 6.7 & 120 & 63 \\
\hline & $4 I-58$ & & $4.6-11$ & & $77-160$ & \\
\hline \multirow[t]{2}{*}{ pol3-0I } & 190 & 43 & 19 & 15 & 110 & 59 \\
\hline & $136-260$ & & $6-35$ & & $80-160$ & \\
\hline \multirow[t]{2}{*}{ pol3-0I poll-Y869A } & 220 & 50 & 34 & 26 & 114 & 60 \\
\hline & $140-240$ & & $27-48$ & & $87-210$ & \\
\hline \multirow[t]{2}{*}{ pol3-Y708A } & 28 & 6.3 & 6.1 & 4.7 & 6.0 & 3.1 \\
\hline & $14-32$ & & $5.0-7.7$ & & $4.8-11$ & \\
\hline \multirow[t]{2}{*}{ pol2-4 pol3-Y708A } & 92 & 21 & 15 & 12 & 73 & 38 \\
\hline & $73-120$ & & $14-18$ & & $61-104$ & \\
\hline
\end{tabular}

pol3-Y708A was combined with the pol2-4, the double pol3-Y708A pol2-4 mutant showed clear multiplicity for forward mutations at the CAN1 locus - a 21-fold increase for the pol2-4 pol3-Y708A double mutant compared to a 3.8-fold increase for pol2-4 and a 6.3-fold increase for pol3-Y708A (Table 5). However, the relationship at the two reversion loci was closer to additivity. This suggests that some but not all errors arising in the pol3-Y708A mutant strain may be proofread by the intrinsic exonuclease activity of Pol $\varepsilon$. Other explanations are also considered below.

\section{Discussion}

Investigation of the mechanism of the mutator phenotype of the yeast Y831A DNA Pol $\varepsilon$ mutant began with the observation that, for an equivalent amount of polymerization activity, the purified Y831A enzyme has an increased ability to convert dNTPs to dNMPs relative to wild-type Pol $\varepsilon$ (Figure 2). The replacement of alanine for a tyrosine in the polymerase active site that is conserved in the $\mathrm{B}$ family of DNA polymerases alters the relative activities of the polymerase and exonuclease active sites of Pol $\varepsilon$. The mutator effect of such mutations is unexpected, since in classical studies with T4 DNA polymerase, mutants that lead to increased nucleotide turnover were antimutators (see [33]).

As the Y831A amino acid replacement is in the polymerase active site rather than the exonuclease active site, it seems likely that the altered protein is somehow compromised in its ability to extend primers, perhaps reflecting reduced catalytic efficiency or increased dissociation from DNA. Any polymerization defect resulting from the alanine substitution is apparently not severe enough to affect the essential role of Pol $\varepsilon$ in cell viability, because a haploid yeast strain containing the pol2-Y831A mutation has normal growth characteristics [7]. This viability provided the opportunity, and the increased dNTP turnover provided the motivation, to investigate further the role of Pol $\varepsilon$ and its proofreading activity in vivo by looking at the combined effects of polymerase and exonuclease active site mutations on mutation rates. These experiments lead to several interpretations.

The relative increase in dNTP turnover suggests altered communication between the polymerase and exonuclease. This may be relevant to the frameshift mutagenesis promoted by the pol2-Y831A mutation (Tables 1,2,3,4 and [7]). For example, misaligned intermediates may form as the primer partitions between double-stranded DNA in the polymerase active site and single-stranded DNA in the exonuclease active site (for example, see [3436]).

The fact that pol2-Y831 A suppresses mutations resulting from inactivation of proofreading by Pol $\varepsilon$ by the pol2-4 mutation (Tables 1, 2 and 3 ) suggests limited involvement of Y831A Pol $\varepsilon$ in DNA synthesis in vivo. This is con- 
sistent with the turnover data and the idea that the Y831A change compromises the ability of Pol $\varepsilon$ to extend primers. On the other hand, the pol2-Y831A mutation does not lead to any obvious effects on growth. In this respect, our results are different from the study of a pol3- $t$ mutation in the POL3 gene encoding catalytic subunit of Pol $\delta$. The pol3- $t$ also suppresses the mutator effect of the pol3-01 exonuclease defective allele in a strain with a double mutation but causes a temperature-sensitive growth phenotype [37]. One possibility that explains our data is increased polymerase dissociation from the primer-template. Increased dissociation has been proposed earlier to explain observations with mutant forms of two other replicative polymerases, Escherichia coli DNA polymerase III [38] and bacteriophage RB69 DNA polymerase [39]. For example, when wild-type Pol $\varepsilon$ misinserts a nucleotide, the major pathway of error avoidance is exonucleolytic proofreading (Figure 3A, thick black arrow), whereas the mutation is generated if the mismatch is extended (central arrow). Inactivation of the intrinsic exonuclease of Pol $\varepsilon$, as with the pol2-4 allele, yields substantial mutator effects, suggesting that under normal circumstances, wild-type Pol $\varepsilon$ dissociation to allow mismatch excision by a different exonuclease may be rare (as indicated by the thin arrow). However, it may be that for Y831A Pol $\varepsilon$ (Figure 3A, bottom), the dissociation mechanism prevails (thick arrow) over the proofreading mechanism, such that the mutator effect of a Pol $\varepsilon$ proofreading deficiency is small, as observed here.

An alternative model is based on the hypothesis that Pol $\varepsilon$ has three distinct roles in replication (Figure $3 \mathrm{~B}$ ). We propose that wild-type Pol $\varepsilon$ has a limited DNA synthetic role during S-phase (Figure $3 \mathrm{~B}$, left). Indeed, its in vitro fidelity is distinct from all replicative DNA polymerases studied so far [40]. Since the mutator effect of the pol2-4 is approximately 10-fold lower that that of the pol3-01, we can tentatively estimate that Pol $\varepsilon$ would catalyze less than $10 \%$ of the bulk DNA synthesis during replication (indicated by thin arrow). This calculation is made under the assumption that Pol $\varepsilon$ and Pol $\delta$ have similar base selectivity (currently under investigation). However, Pol $\varepsilon$ may have more substantial roles in replicating certain regions/ sites in the genome (arrow in the center), perhaps where proofreading is less active (for example, long homopolymeric runs, see [41]), and in performing limited reactions that are important for vegetative growth, for example, initiation of DNA replication [24]). This hypothesis is consistent with the genetic interactions of the pol2-4 and pol2Y831A combinations in cis and trans (Tables 1,2,3). In this model, the mutator effect of the pol2-4 allele results from Pol $\varepsilon$ involvement in general S-phase replication (Figure 3B, left). The Y831A amino acid change alters the enzyme as discussed above, reducing its involvement in general Sphase replication (indicated by a dashed thin arrow), but permits its functions in the two other pathways, albeit with reduced fidelity (Figure $3 \mathrm{~B}$, right). The mutator effects of the pol2-Y831A might then reflect inaccurate replication at specific sites, or general perturbation of replication, rationalizing the lack of synergistic increases in mutation rates at some loci in strains with the pol2-4 (Table 1) or pol3-01 (Table 4) alleles and, on the other hand, semi-dominant mutator effect of pol2-Y831A in diploids when another allele is wild-type POL2. Alternatively or additionally, altered replication efficiency or fidelity in strains harboring mutant polymerase alleles may lead to activation of a checkpoint response that results in the accumulation of mutations [42].

The model in Figure 3B can also explain the increases in mutagenesis in the pol2-4 pol1-Y869A and pol2-4 pol3Y708A mutant strains (Table 5), by invoking increased DNA synthesized by Pol $\varepsilon$ during S-phase when Pol $\alpha$ or Pol $\delta$ is impaired. Especially interesting here are the synergistic increases in mutation rates at the CAN1 locus. This synergy suggests that under certain circumstances, at least some errors generated by Pol $\alpha$ and Pol $\delta$ may be proofread by the intrinsic exonuclease activity of Pol $\varepsilon$. In the future, it will be interesting to test this hypothesis further, to determine under what circumstances, for example, translesion DNA synthesis [43] or base excision repair [44] errors made by one DNA polymerase may be proofread by an exonuclease intrinsic to a second DNA polymerase or even another protein [45].

\section{Conclusions}

Y831A replacement reduces replication fidelity and participation of Pol $\varepsilon$ in chromosomal replication, but without eliminating an additional function of Pol $\varepsilon$ that is essential for viability. This suggests that other polymerases can substitute for certain functions of Pol $\varepsilon$. Conversely, Pol $\varepsilon$ can proofread errors made by polymerases $\alpha$ and $\delta$.

\section{Methods \\ Strains}

For mutant construction we used strains 8C-YUNI101 and E134 described earlier [7]. Y831A DNA polymerase $\varepsilon$ holoenzyme was purified from the protease-deficient strain ep831-T334 (MAT $\alpha$ pol2-Y831A pep4-3 prb1-1122 reg1-501 gal1 ura3-52 leu2-3,112 trp1::hisG can1 LYS2). To construct the strain, we introduced the pol2-Y831A allele into the strain T334 [46], a Trp- derivative of 334 described in [47], by a method described earlier [7].

\section{Construction of mutants with DNA polymerase mutations} All mutations were introduced into chromosomal DNA polymerase genes by the integration-excision method described earlier $[7,18]$. Double mutants were constructed sequentially. To construct a double mutation in the POL2 gene, we transformed a strain already possessing pol2- 


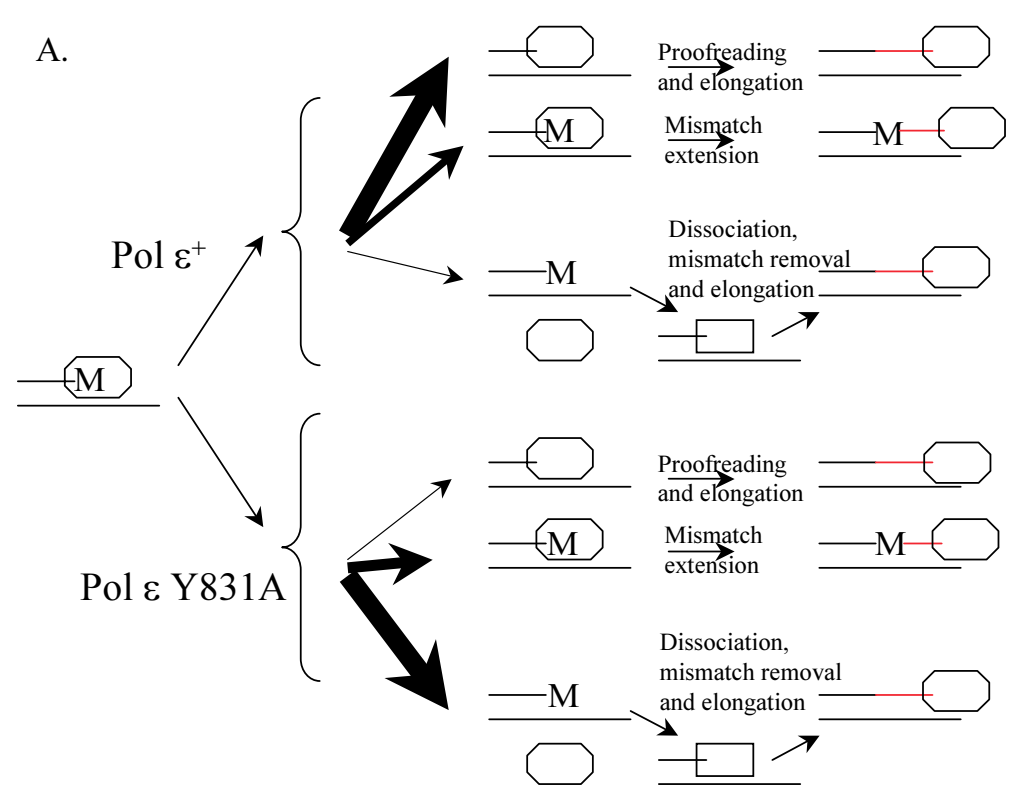

B.
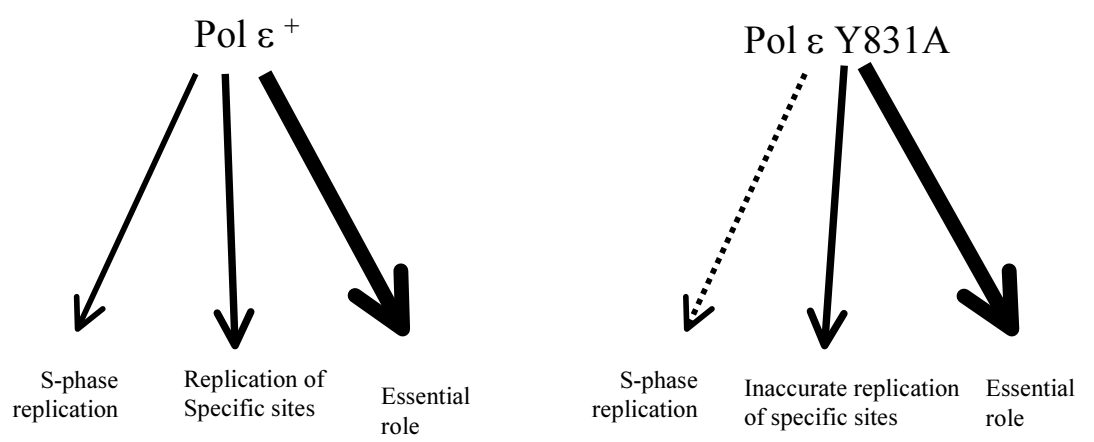

Figure 3

Models explaining results on the genetic interaction of mutants with defects in the active site and proofreading of DNA polymerase $\varepsilon$. Thickness of arrows indicates the relative probability of a depicted pathway. See text for explanations. (A) Dissociation-proofreading. The newly synthesized DNA is in red. 'M' stands for incorrectly inserted nucleotide. DNA polymerase is drawn as octagonal oval; an exonuclease is drawn as rectangle. (B) Three distinct roles of Pol $\varepsilon$ in replication. 
Y831A to Ura ${ }^{+}$with the plasmid pJB1 (carrying the pol2-4 mutation) cut by BamHI. Transformants were plated on a medium with 5-fluoroorotic acid to select for Ura- clones resulting from the loss of the plasmid. Resulting clones were tested by PCR for the presence of both mutations. Detection of pol2-Y831A was described previously [7]. To detect the pol2-4 mutation, we amplified a 481 bp region of the POL2 gene using primers M32-m, 5'TCCGAGTATCTATAGACAAGGA and M36, 5'CTCACCTTCAGCATCTGG. The resulting fragment was digested with $S f c I$. The presence of the pol2-4 mutation creates a new $S f c$ I site; the PCR fragment is cut into 119 and 362 base pair fragments. The same mutations were created in basic haploid strains 8C-YUNI101 and E134. The resulting mutants were then crossed to generate diploids homozygous or heterozygous for polymerase mutations.

\section{Mutation rate measurements}

Measurements of mutation rates were performed as described [7].

\section{Purification of DNA polymerase $\varepsilon$}

The four-subunit DNA polymerase $\varepsilon$ was purified from 1.2 $\mathrm{kg}$ of the protease deficient ep831-T334 strain as described in $[23,29]$ with modifications. Briefly, after an ammonium sulfate precipitation step and SP-Sepharose chromatography $[23,29]$, the dialyzed sample was loaded onto a Q Sepharose HP60/100 column (Amersham Biosciences Corp., Piscataway, NJ, USA) and eluted with nine column volumes of a linear gradient of $\mathrm{NaCl}$ from 50 to $500 \mathrm{mM}$. Four peaks with DNA polymerase activity were obtained, and the peak containing the four-subunit Pol $\varepsilon$ was identified by immunoblotting with polyclonal antibodies [29]. Further fractionation of pooled fractions from this peak by MonoS H/R and HiTrap heparin columns (Amersham Biosciences) was performed as described [23]. Finally, fractions with DNA polymerase $\varepsilon$ from the Heparin column were loaded onto a MonoS 1.6/ 5 column in a SMART system (Amersham Biosciences). The polymerase was eluted by a linear $\mathrm{NaCl}$ gradient from 50 to $1000 \mathrm{mM}$. Fractions $(30 \mu \mathrm{l})$ were collected and assayed for activity. One UV absorbance peak was observed and it coincided with one peak of polymerase activity. The purity of the preparation was approximately $90 \%$ as judged from SDS-PAGE electrophoresis and SimpleStain (Invitrogen, Carlsbad, California, USA) colloidal coumassie staining (Figure 1). Peak fractions with specific polymerase activity around $4,000 \mathrm{U} / \mathrm{mg}$ were pooled and stored at $-80^{\circ} \mathrm{C}$.

\section{Enzyme activity determination}

Polymerase activity was determined using a poly $(\mathrm{dA})_{300} /$ oligo $(\mathrm{dT})_{10}$ substrate as described [23]. Reaction mixtures $(20 \mu \mathrm{l})$ were incubated at $30^{\circ} \mathrm{C}$, and $5 \mu \mathrm{l}$ aliquots were withdrawn at the indicated time intervals and placed in tubes with $1 \mu \mathrm{l}$ of $300 \mathrm{mM}$ EDTA. One $\mu \mathrm{l}$ of these mixtures was spotted onto each of the two PEI (Merck, Whitehouse Station, NJ, USA) plates. One was developed in $1 \mathrm{M} \mathrm{LiCl}$, $1 \mathrm{M}$ formic acid, $50 \mathrm{mM} \mathrm{NaH}_{2} \mathrm{PO}_{4}$, thus separating the unincorporated label from label incorporated into DNA. A second plate was developed with essentially the same solution but containing only $0.4 \mathrm{M} \mathrm{LiCl}$. This permits quantification of the amount of labeled nucleoside monophosphate generated (that is, turnover of dNTP by the DNA polymerase-associated proofreading exonuclease, see Figure 2A,2B). The intensity of the spots was quantified by BAS2500 Bio Image Analyzer (Fuji Photo Film Company, Tokyo, Japan).

Exonuclease assays employed a 3'-radiolabelled poly $(\mathrm{dA})_{300}$ single-stranded substrate. Three $\mu \mathrm{g}$ of poly $(\mathrm{dA})_{300}$ were labeled by terminal transferase with 40 $\mu \mathrm{Ci}$ of $\alpha^{-32} \mathrm{P}$ deoxyadenosine triphosphate (dATP) in 1 mM dATP and 1x One-for-All Plus buffer (Promega, Madison, WI, USA). Reaction products were purified using a Qiagen nucleotide removal kit (Qiagen, Valencia, CA, USA) and then examined by TLC. An average of nine dATP molecules were added per each poly $(\mathrm{dA})_{300}$ molecule. Exonuclease reactions $(20 \mu \mathrm{l})$ with $37 \mathrm{ng}(0.14 \mu \mathrm{Ci})$ of labeled substrate and $200 \mathrm{ng}$ of unlabeled poly $(\mathrm{dA})_{300}$ were performed in the same buffer as the polymerase reaction, also at $30^{\circ} \mathrm{C}$. At each time point, one $\mu$ l of reaction mixture was spotted onto a PEI plate and developed with $0.4 \mathrm{M} \mathrm{LiCl}$ as described above. The polymerase activity estimate was compensated by dividing the apparent value of incorporation (acid insoluble nucleotides) with 0.78 for the wild-type enzyme (turnover rate $=22 \%$ ) and 0.50 for the mutant enzyme (turnover rate $=50 \%$ ).

\section{Authors' contributions}

YIP and SM purified and did initial characterization of polymerase $\varepsilon$ Y831A. SM purified wild-type enzyme and performed most of the biochemical characterization of the enzymes. YIP did genetic experiments with yeast. YIP, SM, HM and TK participated in the design of the study. TK coordinated the study. All authors read and approved the final manuscript.

\section{Acknowledgements}

We would like to thank Drs A Sugino and $\mathrm{H}$ Araki for valuable comments throughout the course of this study, and PV Shcherbakova and R Kokoska for critical reading of the manuscript.

\section{References}

I. Sugino A: Yeast DNA polymerases and their role at the replication fork. Trends Biochem Sci 1995, 20:319-323.

2. Burgers PM: Eukaryotic DNA polymerases in DNA replication and DNA repair. Chromosoma 1998, 107:218-227.

3. Goodman MF: Error-prone repair DNA polymerases in prokaryotes and eukaryotes. Annu Rev Biochem 2002, 71:17-50.

4. Shcherbakova PV, Bebenek K, Kunkel TA: Functions of eukaryotic DNA polymerases. 2003, 2003: [http://sageke.sciencemag.org/cgi/ content/fulltext/2003/8/re3]. Science's SAGE KE 
5. Lawrence CW: DNA polymerase $\zeta$ and the control of DNA damage induced mutagenesis in eukaryotes. Genetic instability in cancer Volume 28. Imperial Cancer Research Fund; 1996:2I-3I.

6. Morrison A, Sugino A: The 3'-->5' exonucleases of both DNA polymerases delta and epsilon participate in correcting errors of DNA replication in Saccharomyces cerevisiae. Mo Gen Genet 1994, 242:289-296.

7. Pavlov YI, Shcherbakova PV, Kunkel TA: In vivo consequences of putative active site mutations in yeast DNA polymerases alpha, epsilon, delta, and zeta. Genetics 200I, 159:47-64.

8. Prakash S, Prakash L: Translesion DNA synthesis in eukaryotes: a one- or two-polymerase affair. Genes Dev 2002, 16:1872-1883.

9. Rattray AJ, Strathern JN: ERROR-PRONE DNA POLYMERASES: When Making a Mistake is the Only Way to Get Ahead I. Annu Rev Genet 2003, 37:31-66.

10. Hile SE, Eckert KA: Positive correlation between DNA polymerase alpha-primase pausing and mutagenesis within polypyrimidine/polypurine microsatellite sequences. J Mol Biol 2004, 335:745-759.

II. Tsubota T, Maki S, Kubota H, Sugino A, Maki H: Double-stranded DNA binding properties of Saccharomyces cerevisiae DNA polymerase epsilon and of the Dpb3p-Dpb4p subassembly. Genes Cells 2003, 8:873-888.

12. Johansson E, Garg P, Burgers PM: The Pol32 subunit of DNA polymerase delta contains separable domains for processive replication and proliferating cell nuclear antigen (PCNA) binding. J Biol Chem 2004, 279: I907-1915.

13. Morrison A, Araki H, Clark AB, Hamatake RK, Sugino A: A third essential DNA polymerase in $\mathbf{S}$. cerevisiae. Cell 1990 , 62: I|43-|| I5I.

14. Burgers PM: Saccharomyces cerevisiae replication factor C. II. Formation and activity of complexes with the proliferating cell nuclear antigen and with DNA polymerases delta and epsilon. I Biol Chem 1991, 266:22698-22706.

15. Shcherbakova PV, Pavlov YI: 3'-->5' exonucleases of DNA polymerases epsilon and delta correct base analog induced DNA replication errors on opposite DNA strands in Saccharomyces cerevisiae. Genetics 1996, I42:717-726.

16. Karthikeyan R, Vonarx EJ, Straffon AF, Simon M, Faye G, Kunz BA Evidence from mutational specificity studies that yeast DNA polymerases delta and epsilon replicate different DNA strands at an intracellular replication fork. J Mol Biol 2000, 299:405-419.

17. Fukui T, Yamauchi K, Muroya T, Akiyama M, Maki H, Sugino A, Waga $S$ : Distinct roles of DNA polymerases delta and epsilon at the replication fork in Xenopus egg extracts. Genes Cells 2004, 9:179-191.

18. Morrison A, Bell JB, Kunkel TA, Sugino A: Eukaryotic DNA polymerase amino acid sequence required for $3^{\prime}->5$ ' exonuclease activity. Proc Natl Acad Sci USA 199I, 88:9473-9477.

19. Morrison A, Johnston AL, Johnston LH, Sugino A: Pathway correcting DNA replication errors in S. cerevisiae. EMBO J 1993, I 2: |467-|473

20. Kesti T, Flick K, Keranen S, Syvaoja JE, Wittenberg C: DNA polymerase epsilon catalytic domains are dispensable for DNA replication, DNA repair, and cell viability. Mol Cell 1999 , 3:679-685.

21. Ohya T, Kawasaki Y, Hiraga S, Kanbara S, Nakajo K, Nakashima N, Suzuki A, Sugino A: The DNA polymerase domain of pol(epsilon) is required for rapid, efficient, and highly accurate chromosomal DNA replication, telomere length maintenance, and normal cell senescence in Saccharomyces cerevisiae. Biol Chem 2002, 277:28099-28I08.

22. Navas TA, Zhou Z, Elledge SJ: DNA polymerase epsilon links the DNA replication machinery to the $S$ phase checkpoint. Cell 1995, 80:29-39.

23. Maki S, Hashimoto K, Ohara T, Sugino A: DNA polymerase II (epsilon) of Saccharomyces cerevisiae dissociates from the DNA template by sensing single-stranded DNA. J Biol Chem 1998, 273:21332-2134|.

24. Masumoto $H$, Sugino A, Araki H: Dpb I I controls the association between DNA polymerases alpha and epsilon and the autonomously replicating sequence region of budding yeast. Mol Cell Biol 2000, 20:2809-28I7.

25. Takayama Y, Kamimura Y, Okawa M, Muramatsu S, Sugino A, Araki $\mathrm{H}$ : GINS, a novel multiprotein complex required for chromo- somal DNA replication in budding yeast. Genes Dev 2003, I7: II53-II65.

26. Fuss J, Linn S: Human DNA polymerase epsilon colocalizes with proliferating cell nuclear antigen and DNA replication late, but not early, in S phase. J Biol Chem 2002, 277:8658-8666.

27. Edwards S, Li CM, Levy DL, Brown J, Snow PM, Campbell JL: Saccharomyces cerevisiae DNA polymerase epsilon and polymerase sigma interact physically and functionally, suggesting a role for polymerase epsilon in sister chromatid cohesion. Mol Cell Biol 2003, 23:2733-2748.

28. Morrison A, Sugino A: Roles of POL3, POL2 and PMSI genes in maintaining accurate DNA replication. Chromosoma 1992 102:SI47-9.

29. Hamatake RK, Hasegawa H, Clark AB, Bebenek K, Kunkel TA, Sugino A: Purification and characterization of DNA polymerase II from the yeast Saccharomyces cerevisiae. Identification of the catalytic core and a possible holoenzyme form of the enzyme. J Biol Chem 1990, 265:4072-4083.

30. Shimizu K, Hashimoto K, Kirchner JM, Nakai W, Nishikawa H, Resnick MA, Sugino A: Fidelity of DNA polymerase epsilon holoenzyme from budding yeast Saccharomyces cerevisiae. J Biol Chem 2002, 277:37422-37429.

31. Shcherbakova PV, Kunkel TA: Mutator phenotypes conferred by MLHI overexpression and by heterozygosity for $\mathrm{mlhI}$ mutations. Mol Cell Biol 1999, 19:3177-3183.

32. Perrino FW, Loeb LA: Hydrolysis of 3 '-terminal mispairs in vitro by the 3 ' to 5 ' exonuclease of DNA polymerase delta permits subsequent extension by DNA polymerase alpha. Biochemistry 1990, 29:5226-5231.

33. Nossal NG: A new look at old mutants of T4 DNA polymerase. Genetics 1998, I48:1535-1538.

34. Bebenek K, Kunkel TA: Frameshift errors initiated by nucleotide misincorporation. Proc Natl Acad Sci U S A 1990, 87:4946-4950.

35. Seki M, Akiyama M, Sugaya Y, Ohtsubo E, Maki H: Strand asymmetry of + I frameshift mutagenesis at a homopolymeric run by DNA polymerase III holoenzyme of Escherichia coli. J Biol Chem 1999, 274:33313-33319.

36. Sugaya $Y$, Ihara K, Masuda Y, Ohtsubo E, Maki H: Hyper-processive and slower DNA chain elongation catalysed by DNA polymerase III holoenzyme purified from the dnaEI 73 mutator mutant of Escherichia coli. Genes Cells 2002, 7:385-399.

37. Tran HT, Degtyareva NP, Gordenin DA, Resnick MA: Genetic factors affecting the impact of DNA polymerase delta proofreading activity on mutation avoidance in yeast. Genetics 1999 , I 52:47-59.

38. Fijalkowska IJ, Schaaper RM: Effects of Escherichia coli dnaE antimutator alleles in a proofreading-deficient mutD5 strain. J Bacteriol 1995, I 77:5979-5986.

39. Bebenek A, Dressman HK, Carver GT, Ng S, Petrov V, Yang G, Konigsberg WH, Karam JD, Drake JW: Interacting fidelity defects in the replicative DNA polymerase of bacteriophage RB69.J Biol Chem 200I, 276: I0387-10397.

40. Shcherbakova PV, Pavlov YI, Chilkova O, Rogozin IB, Johansson E, Kunkel TA: Unique error signature of the four-subunit yeast DNA polymerase epsilon. J Biol Chem 2003, 278:43770-43780.

4I. Tran HT, Keen JD, Kricker M, Resnick MA, Gordenin DA: Hypermutability of homonucleotide runs in mismatch repair and DNA polymerase proofreading yeast mutants. Mol Cell Biol 1997, 17:2859-2865

42. Datta A, Schmeits JL, Amin NS, Lau PJ, Myung K, Kolodner RD: Checkpoint-dependent activation of mutagenic repair in Saccharomyces cerevisiae pol3-0I mutants [In Process Citation]. Mol Cell 2000, 6:593-603.

43. Bebenek K, Matsuda T, Masutani C, Hanaoka F, Kunkel TA: Proofreading of DNA polymerase eta-dependent replication errors. Biol Chem 200I, 276:2317-2320.

44. Matsuda T, Vande Berg BJ, Bebenek K, Osheroff WP, Wilson SH, Kunkel TA: The base substitution fidelity of DNA polymerase beta-dependent single-nucleotide base excision repair. I Biol Chem 2003, 278:25947-2595।.

45. Chou KM, Cheng YC: An exonucleolytic activity of human apurinic/apyrimidinic endonuclease on 3' mispaired DNA. Nature 2002, 415:655-659.

46. Lewis LK, Westmoreland JW, Resnick MA: Repair of endonuclease-induced double-strand breaks in Saccharomyces cerevi- 
siae: essential role for genes associated with nonhomologous end-joining. Genetics 1999, I52:1513-1529.

47. Hovland P, Flick J, Johnston M, Sclafani RA: Galactose as a gratuitous inducer of GAL gene expression in yeasts growing on glucose. Gene 1989, 83:57-64.

Publish with Biomed Central and every scientist can read your work free of charge

"BioMed Central will be the most significant development for disseminating the results of biomedical research in our lifetime. " Sir Paul Nurse, Cancer Research UK

Your research papers will be:

- available free of charge to the entire biomedical community

- peer reviewed and published immediately upon acceptance

- cited in PubMed and archived on PubMed Central

- yours - you keep the copyright

Submit your manuscript here:

http://www.biomedcentral.com/info/publishing_adv.asp
BiolMedcentral 